This manuscript has been authored by Fermi Research Alliance, LLC under Contract No. DE AC02-07CH11359 with the U.S. Department of

Energy, Office of Science, Office of High Energy

Physics.

\title{
존
}

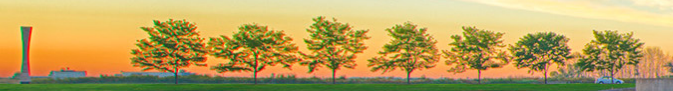

\section{QMiR Crab Cavity for ILC}

Andrei Lunin, Vyacheslav Yakovlev

October 27, 2021

\section{ILC Workshop on Potential Experiments}

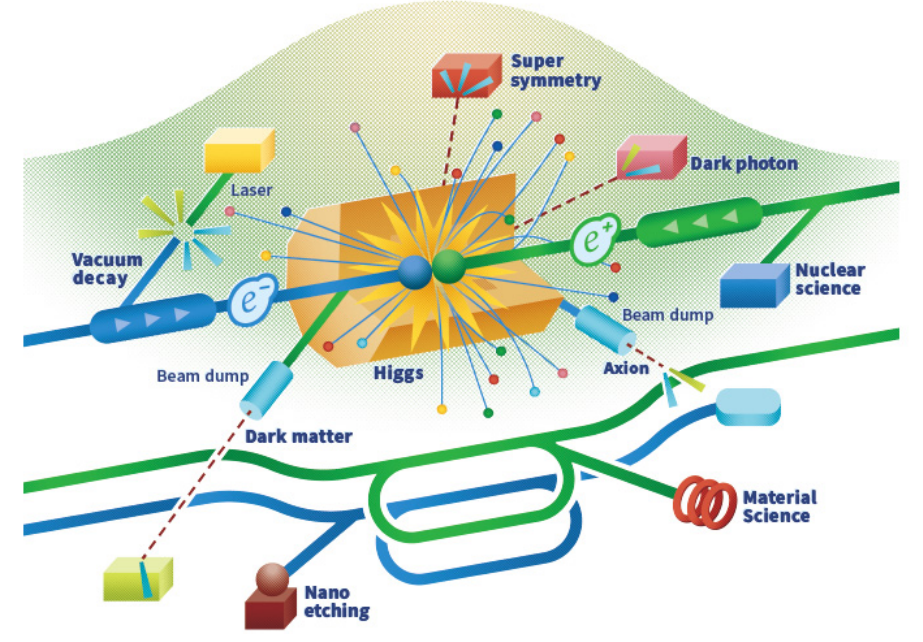




\section{Outline}

- General Requirements for the ILC deflecting cavities

- HOM impedance limitation due to resonance excitation

- Transverse effects

- Single-bunch effects

- QMiR Deflecting Cavity for ANL/SPX

- Conceptions

- Production and Testing

- QMiR (2.6 GHz) scaled version for ILC

- RF Power Requirements

- HOM and Wakefields Analysis

- Mechanical Analysis (LFD and dF/dP)

- Frequency Tuner and Dressed Cavity Design

- Conclusions 


\section{Requirements for the ILC Crab Cavities (CC)}

Crab cavity location ( present ILC optics deck )

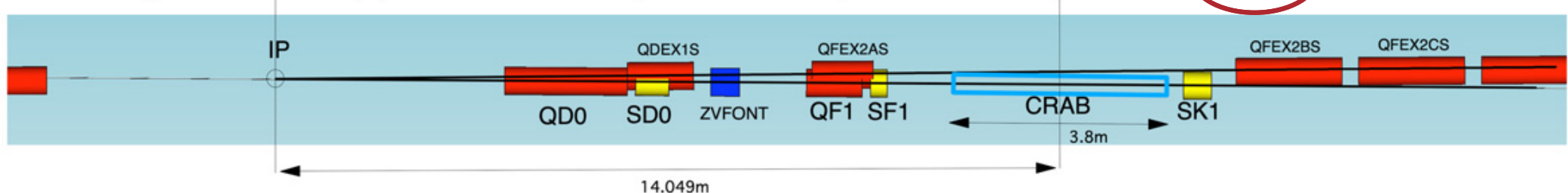

T. Okugi, ILC Crab Specification Final Discussion meeting, 08/08/21

\begin{tabular}{ll}
\hline Beam energy & $E=250 \mathrm{GeV}$ and $\gamma=5 \times 10^{5}$ \\
\hline Beam current (pulsed, average) & $I_{p}=5.8 \mathrm{~mA}, I_{a v}=20 \mu \mathrm{A}$ \\
\hline Pulse width & $\mathrm{t}_{\mathrm{p}}=727 \mu \mathrm{s}$ \\
\hline Beta function at the CC position $(\mathbf{X}, \mathbf{Y})$ & $\beta_{x}=2.3 \times 10^{4} \mathrm{~m}, \beta_{y}=1.5 \times 10^{4} \mathrm{~m}$ \\
\hline Bunch charge & $q=3.2 \mathrm{nC}$ \\
\hline CC kick voltage @2.6GHz & $U_{0}=0.92 \mathrm{MV}$ \\
\hline Normalized emittance $(\mathbf{X}, \mathbf{Y})$ & $\varepsilon_{x}=10 \mu \mathrm{m}, \varepsilon_{y}=35 \mathrm{~nm}$ \\
\hline Beam size at CC location $(\mathbf{X}, \mathbf{Y}, \mathbf{Z})$ & $\sigma_{x}=0.97 \mathrm{~mm}, \sigma_{y}=66 \mu \mathrm{m}, \sigma_{z}=300 \mu \mathrm{m}$
\end{tabular}

- The kick voltage is inverse proportional to frequency $\left(V_{t} \sim f^{-1}\right)$

- The CC space is limited by a close beamlines distance $(<0.2 \mathrm{~m})$

- Small CC aperture $(<10 \mathrm{~mm})$ results in large transverse kick

- Crab cavity @2.6 GHz looks a good compromise 


\section{Crab Cavity HOM Impedance Limits}

Resonant HOM Excitation $\left(\boldsymbol{U}_{\boldsymbol{H} \boldsymbol{}} \boldsymbol{M}=\boldsymbol{k}_{\mathbf{0}} \boldsymbol{x}_{\mathbf{0}} \boldsymbol{I}_{\boldsymbol{p}} \boldsymbol{r}_{\perp}\right)$ can cause:

a) Crabbing voltage distortion

- HOM kick voltage should be less than the crabbing voltage

$$
U_{H O M} \ll U_{0} \sigma_{z} \omega_{R F} / c \text { or } r_{\perp} \ll \frac{U_{0} \sigma_{z} \omega_{R F} / c}{k_{0} x_{0} I_{p}}
$$

b) Beam emittance dilution

- HOM kick should be less than the transverse momentum spread

$$
\boldsymbol{U}_{\text {HOM }} \ll \frac{\sigma_{p_{\perp} c} c}{e}=\frac{p_{\|} c}{e} \sqrt{\frac{\varepsilon}{\gamma \beta}} \quad \text { or } \quad r_{\perp} \ll \frac{E}{k_{0} x_{0} I_{p}} \sqrt{\frac{\varepsilon}{\gamma \beta}}
$$

For max beam offset @CC: $x_{0}<\sigma_{x}$ and $\boldsymbol{y}_{0}<\sigma_{y}$

- Horizontal Shunt Impedance Limit

$r_{x} f_{\text {HOM }} \ll 61 \mathrm{MOhm} \cdot \mathrm{GHz}$

- Vertical Shunt Impedance Limit

$r_{y} f_{\text {ном }} \ll 0.67 \mathrm{MOhm} \cdot \mathrm{GHz}$ 


\section{Crab Cavity Transverse Wakefields Limits}

Incoherent CC excitation (single-bunch effect) can cause:

a) Crabbing voltage distortion

- Transverse kick should be less than the crabbing voltage

$$
U_{k i c k} \ll U_{0} \sigma_{z} \omega_{R F} / c \text { or } k_{\perp} \ll \frac{U_{0} \sigma_{z} \omega_{R F} / c}{q x_{0}}
$$

b) Beam emittance dilution

- Transverse kick should be increase the bunch emittance

$$
U_{k i c k} \ll \frac{\sigma_{p_{\perp}} c}{e}=\frac{p_{\|} c}{e} \sqrt{\frac{\varepsilon}{\gamma \beta}} \text { or } k_{\perp} \ll \frac{E}{q x_{0}} \sqrt{\frac{\varepsilon}{\gamma \beta}}
$$

For max beam offset @CC: $x_{0}<\sigma_{x}$ and $y_{0}<\sigma_{y}$

Horizontal Kick Factor Limit $\quad \boldsymbol{k}_{x} \quad<2.3 \times 10^{3} \mathrm{~V} / \mathrm{pC} / \mathrm{m}$

Vertical Kick Factor Limit $\quad k_{y}<2.5 \times 10^{3} \mathrm{~V} / \mathrm{pC} / \mathrm{m}$ 


\section{HOM-free Deflecting Cavity}

The key idea is based on the formation of TE "ghost" modes

GHOST MODES IN IMPERFEGT WAVEGUIDES

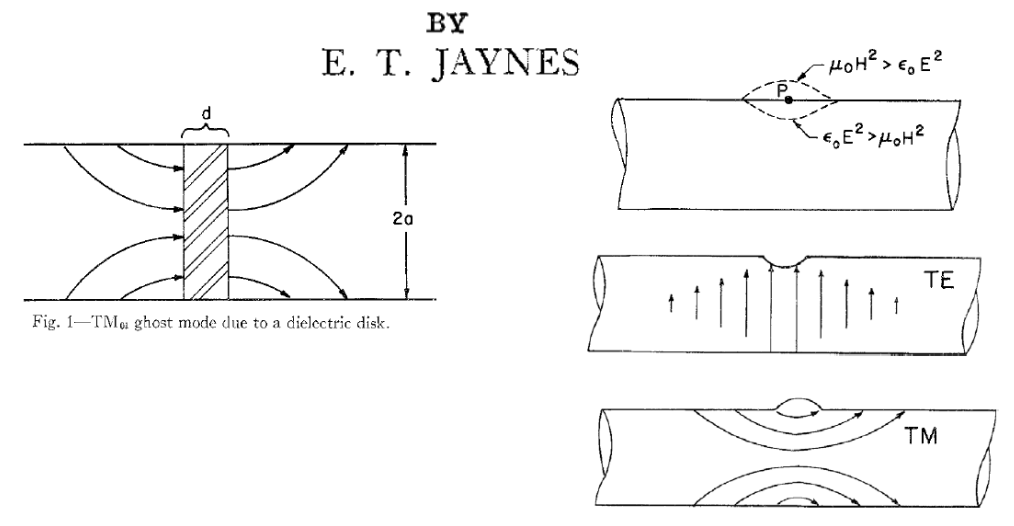

Reprinled from the PROCEEDINGS OF THE IRE VOL. 46, NO. 2, FEBRUARY, 1958

- Rediscovered in 1990s by G. Stupakov and S. Kurennoy [1]

- Further development of RFD at ODU (J. Delayen) [2]

- Transverse kick is produced by Quasi-TE modes which form transition zones with $\operatorname{grad}_{t}\left(E_{z}\right)$

- no contradiction with the Panofscy/Wenzel theorem!

[1] PHYS. REV. VOL. 49-1, 1994

[2] PHYS. REV. SPECIAL TOPICS - ACCELERATORS AND BEAMS 16, 012004 (2013) 


\section{Compact HOM-free Deflecting Cavity QMIR}

\section{Quasi-Waveguide Multicell Deflecting Resonator [1]}

- Proposed as replacement of Mark-II deflecting cavity for APS/SPX project

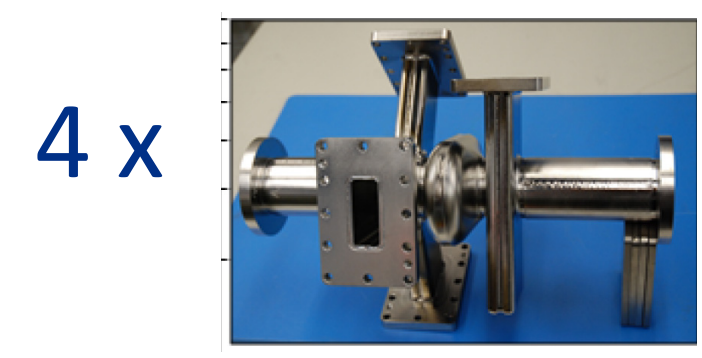

- Prototype built and tested at ANL in 2013 [2]

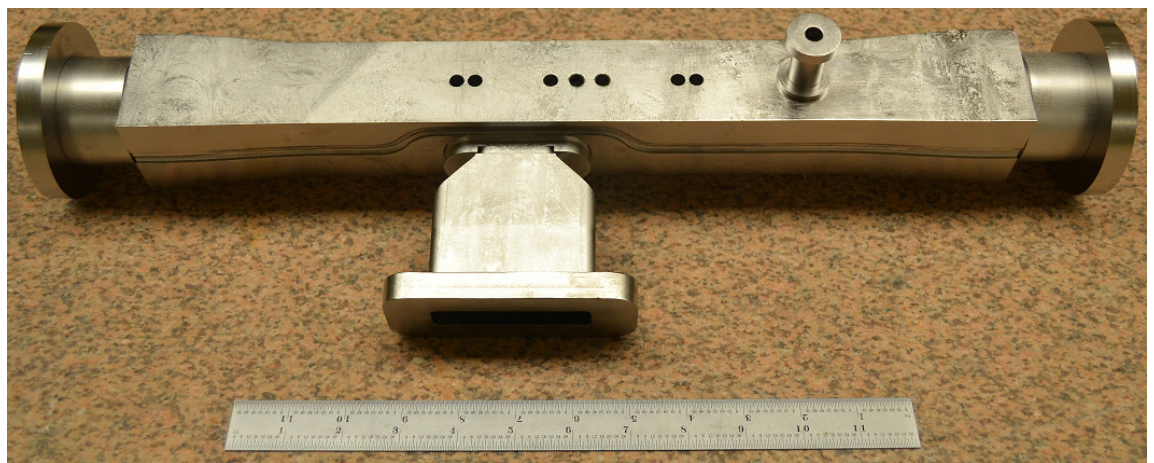

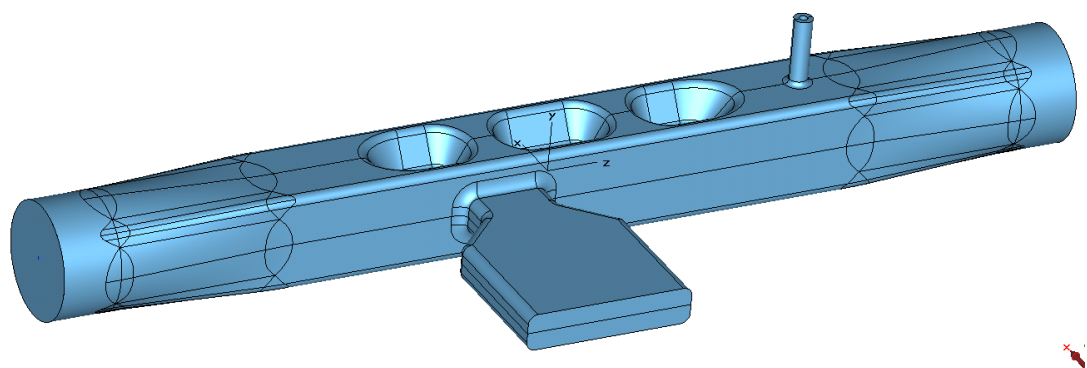

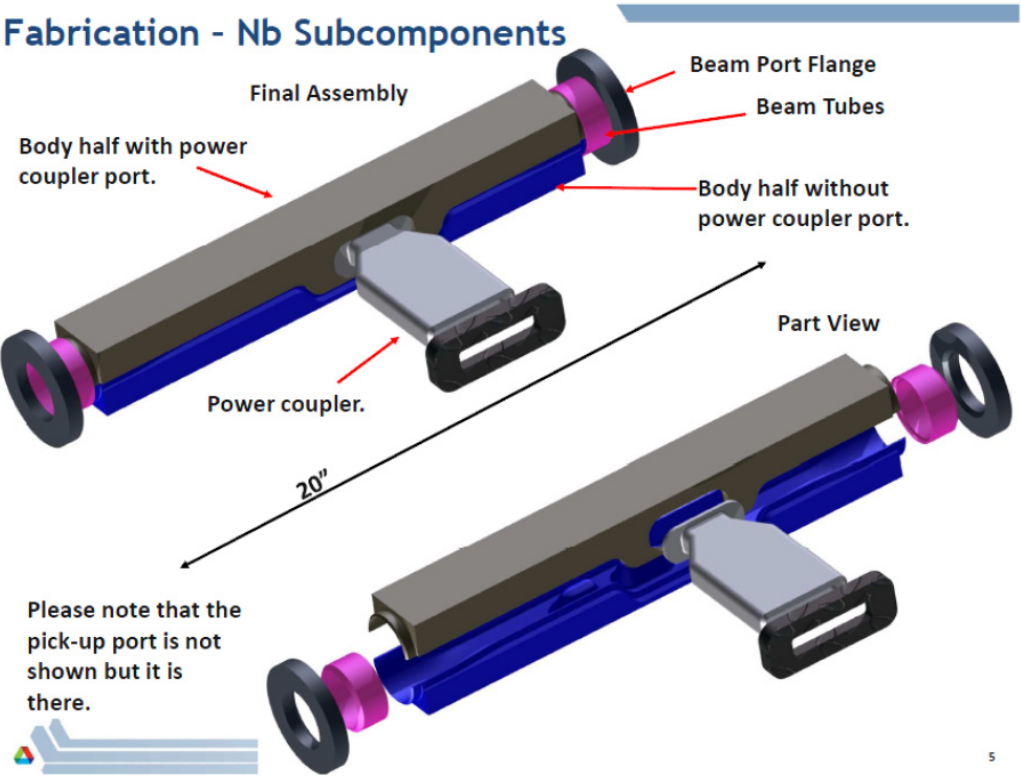

[1] A. Lunin, I. Gonin, M. Awida, T. Khabiboulline, V. Yakovlev, A. Zholents, Physics Procedia 79 ( 2015) $54-62$

[2] Zachary Conway on behalf of ANL PHY LINAC Development Group, 04/23/2013 


\section{QMIR Prototype Production and Testing}

Bare QMiR (21.75' Long) cavity

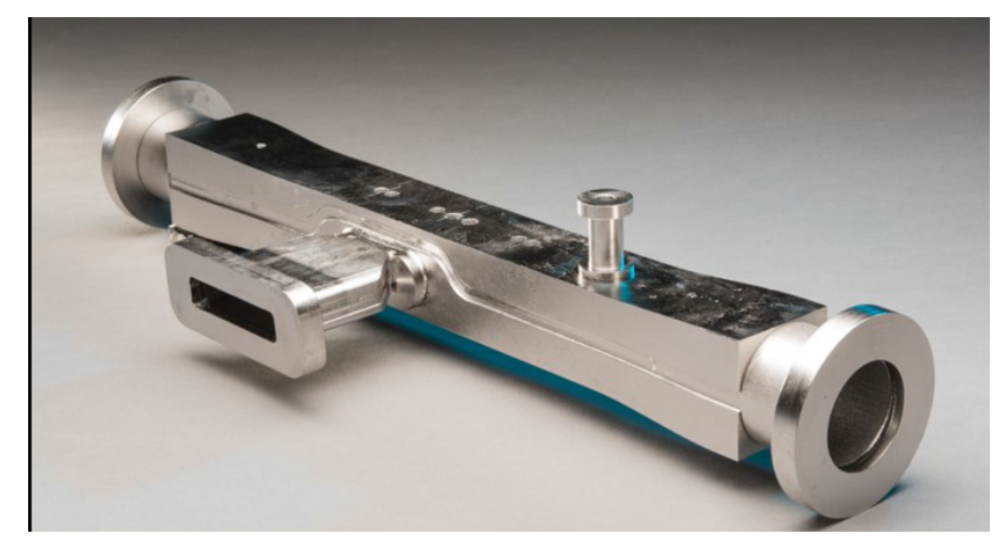

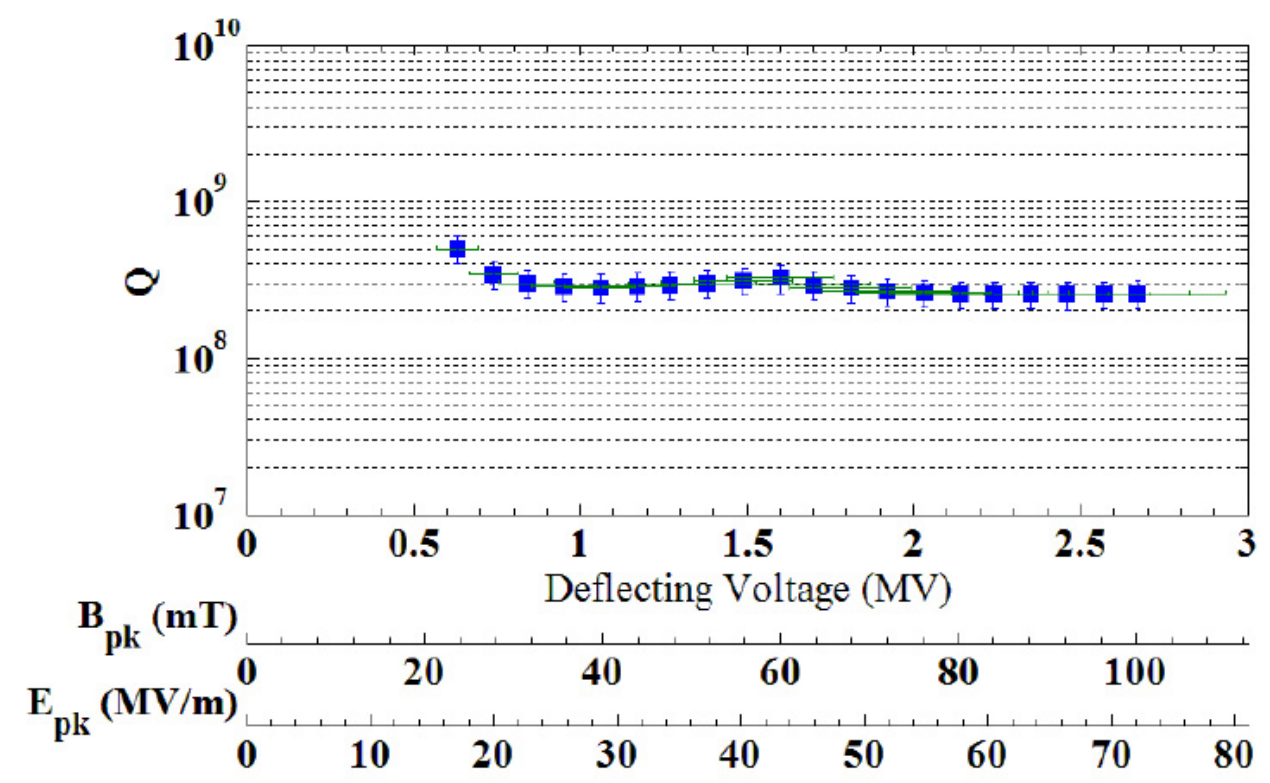

- Cavity received EP-treatment before the test

- Measured maximal deflecting voltage of 2.7 MV exceeded the design goal of 2.0 MV @2K vertical test of QMiR prototype [1]

- Relatively low $Q_{0}(3 E 8)$ is due to extra RF losses at covering flanges

- Further QMiR development was stopped due to the cancelation of ANL/SPX project

Z. Conway, et al., "Development and Test Results of a Quasi-waveguide Multicell Resonator", IPAC14, Dresden, Germany, 2014 苇 Fermilab 


\section{Operating Dipole Mode}

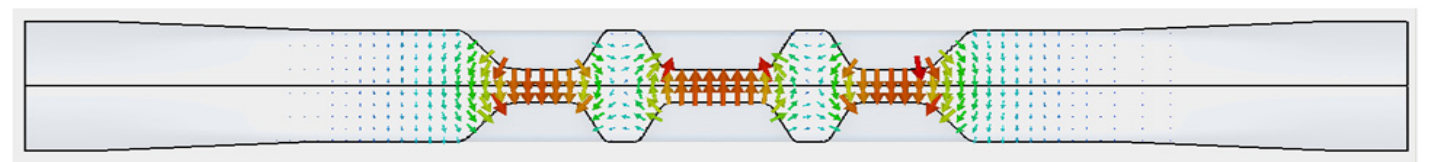

a)

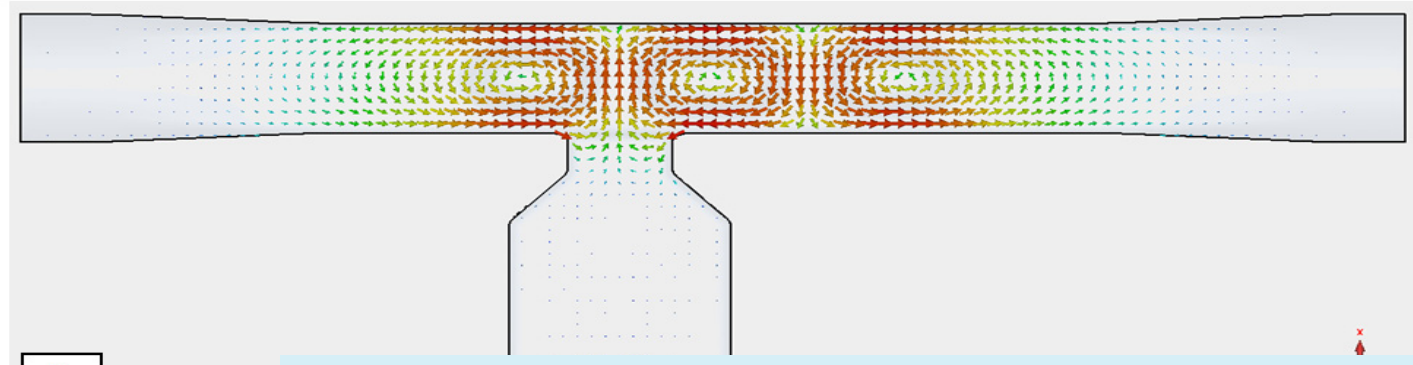

b)

\section{QMiR has a record high $(R / Q)_{t}>1 \mathrm{k} \Omega$}

Transverse electric (blue) and magnetic (red) field components along the cavity axis.

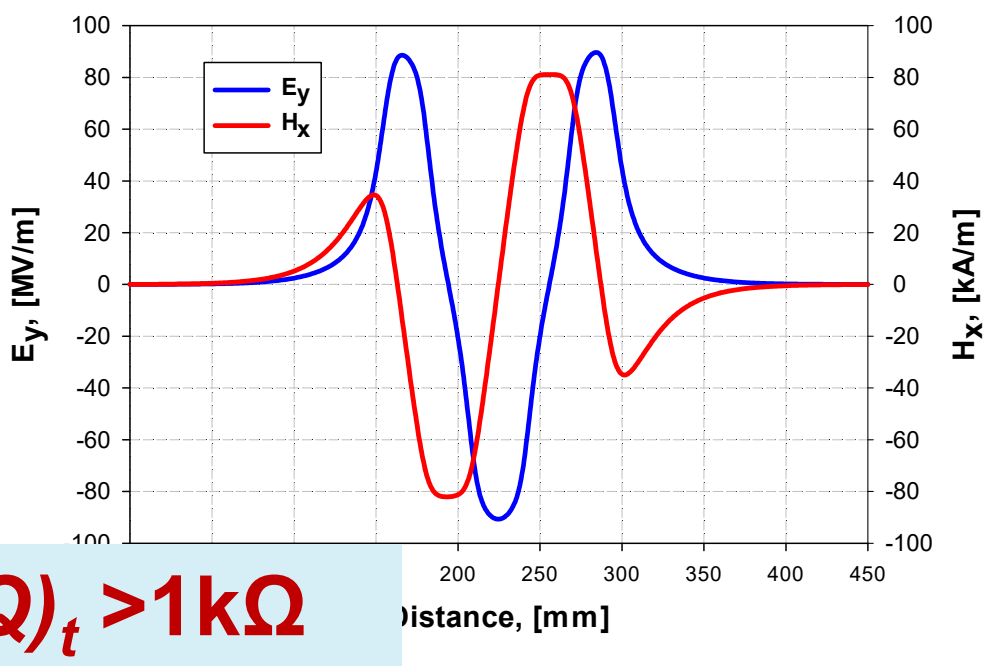

Integrated vertical kick along the cavity axis (solid red curve is the overall kick, dotted blue and green curves are electric and magnetic kicks).

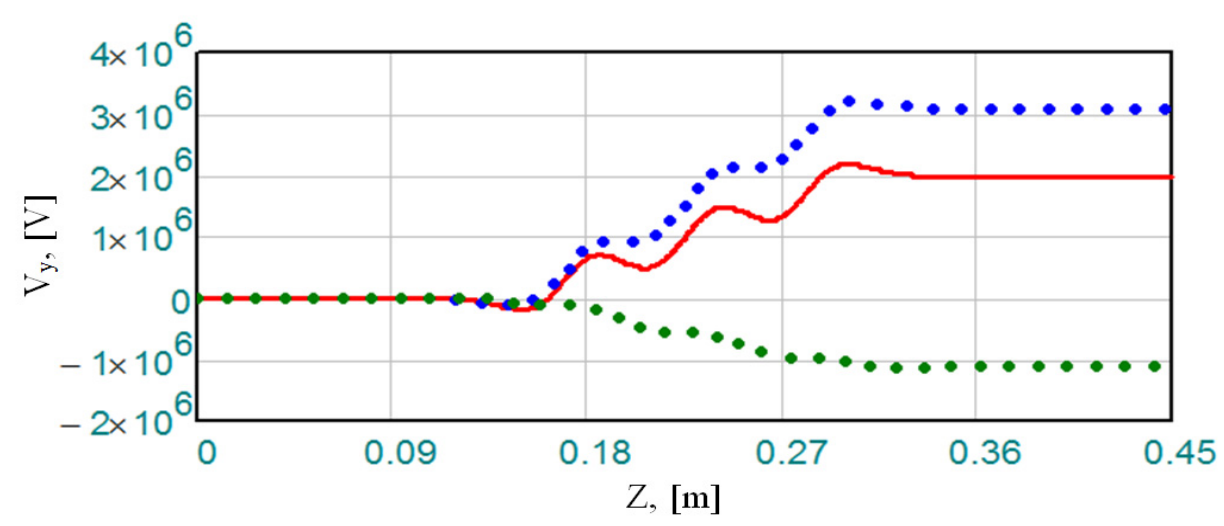

\begin{tabular}{|l|l|}
\hline Freq & $2815 \mathrm{MHz}$ \\
\hline $\mathbf{V}_{\text {kick }}$ & $\mathbf{2} \mathrm{MV}$ \\
\hline $\mathbf{E}_{\max }$ & $\mathbf{5 5 \mathrm { MV } / \mathrm { m }}$ \\
\hline $\mathbf{B}_{\max }$ & $\mathbf{7 6} \mathrm{mT}$ \\
\hline$(\mathbf{R} / \mathbf{Q})_{\mathbf{Y}}$ & $1040 \Omega$ \\
\hline $\mathbf{G}$ & 130 \\
\hline $\mathbf{W}_{\text {STOR }}$ & $\mathbf{0 . 2 3 ~} \mathbf{~}$ \\
\hline
\end{tabular}




\section{HOM Damping in the APS Ring}

\section{QMiR Cavity Monopole HOMs}

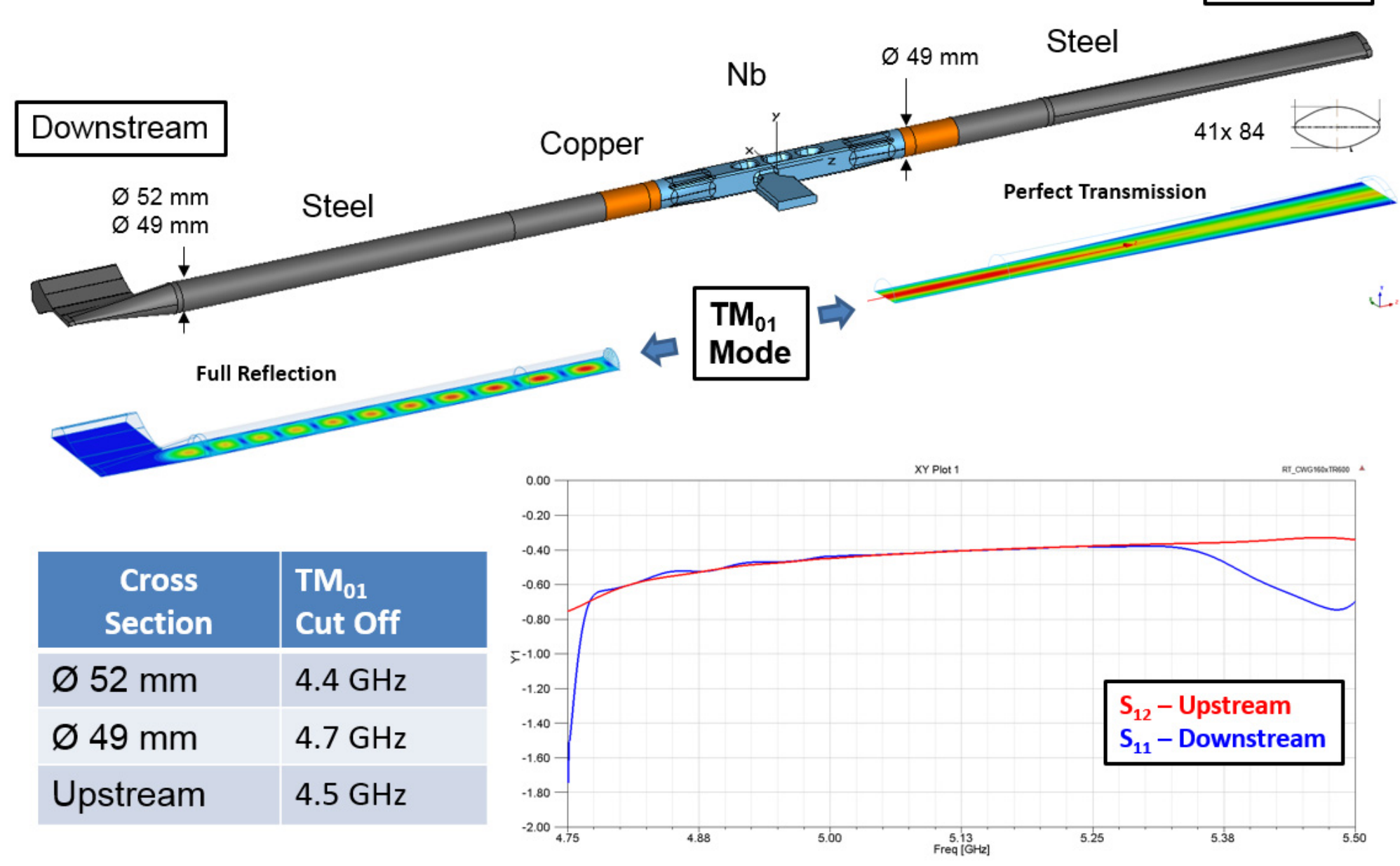

Monopole HOMs RF power is radiated to the Upstream beam pipe ! 


\section{QMiR Cavity for ILC (scaled to $2.6 \mathrm{GHz}$ )}
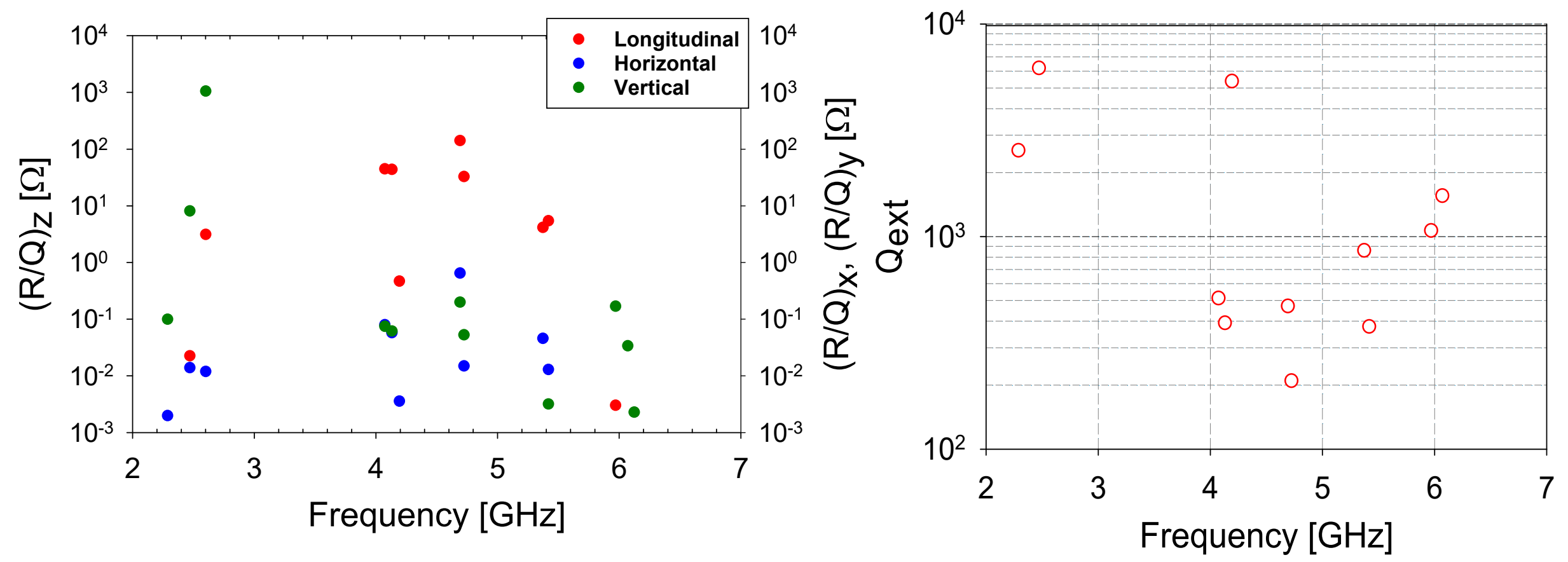

- There are two Same Order Modes (SOM) that have a low $(R / Q){ }^{*} Q$

- SOM/HOM external couplings Qext $<10^{4}$

- SOM/HOMs longitudinal and transverse impedances (@1 $1 \mathrm{~mm}$ ): $(R / Q) z<=100 \Omega,(R / Q) x<=1 \Omega$ and $(R / Q) y<=10 \Omega$

- $\mathrm{SOM} / \mathrm{HOM}$ spectrum is sparse and strongly damped 


\section{QMiR Cavity for ILC (scaled to $2.6 \mathrm{GHz}$ )}

Operation mode

$$
\left(\frac{r_{\perp}}{Q}\right)=1040 \mathrm{Ohm}(@ 2.6 \mathrm{GHz})
$$

Maximal dipole horizontal HOM $\left(\frac{r_{\perp}}{Q}\right)_{x}<10 \mathrm{Ohm}(@ 2.5 \mathrm{GHz})$;

$$
\mathrm{Q}<1 \times 10^{5}\left(<\mathrm{Q}_{\max } \approx 2.4 \times 10^{6}\right)
$$

Maximal dipole vertical HOM

$$
\begin{aligned}
& \left(\frac{r_{\perp}}{Q}\right)_{y}<10 \text { Ohm }(@ 4 \mathrm{GHz}) ; \\
& \mathrm{Q}<1 \times 10^{4}\left(<\mathrm{Q}_{\max } \approx 1.7 \times 10^{6}\right)
\end{aligned}
$$

Incoherent losses

$$
\begin{aligned}
& k_{z} \approx 45 \mathrm{~V} / \mathrm{pC} \\
& P_{\text {rad }} \approx k_{z} q^{2} n_{b} f_{\text {rep }}=3 \mathrm{~W}
\end{aligned}
$$

Horizontal kick factor*

$$
\begin{aligned}
& k_{x}=100(<2300) \mathrm{V} / \mathrm{pC} / \mathrm{m} \\
& k_{y}=400(<2500) \mathrm{V} / \mathrm{pC} / \mathrm{m}
\end{aligned}
$$

* GdfidL calculation for $0.3 \mathrm{~mm}$ bunch length (cross check with ECHO-3D code is ingoing)

- QMiR cavity meets the ILC/CC horizontal and vertical HOM impedance requirements 


\section{QMiR Cavity for ILC RF Power}

- RF power needed to maintain the crabbing voltage should compensate

- the ohmic losses in the cavity (negligible for SRF cavities)

- voltage induced by the beam if the is off the cavity axis

- The maximal required RF power for the detuned cavity:

$$
P=\frac{U_{0}^{2}}{4 Q\left(\frac{r_{\perp}}{Q}\right)}\left[\left(1+\frac{I_{p} Q\left(\frac{r_{\perp}}{Q}\right) k_{0} x_{0}}{U_{0}}\right)^{2}+\left(\frac{2 Q \Delta \omega}{\omega_{0}}\right)^{2}\right]
$$

- For max beam offset $\mathrm{x}_{0}<1 \mathrm{~mm}$ and $\Delta f<1 \mathrm{kHz}$ (LFD, microphonics)

Beam OFF:

Optimal Coupling:

Beam ON \& Microphonics:

$$
\begin{aligned}
& P_{\text {min }} \approx 200 \mathrm{~W} \\
& \mathrm{Q}_{L} \approx 1 \times 10^{6} \\
& P_{\text {max }} \approx 500 \mathrm{~W}
\end{aligned}
$$

- Required RF power from the generator (overhead 100\%):

$$
P_{\text {gen }}<1 \mathrm{~kW}
$$




\section{Mechanical Analysis LFD and dF/dP (by I. Gonin)}

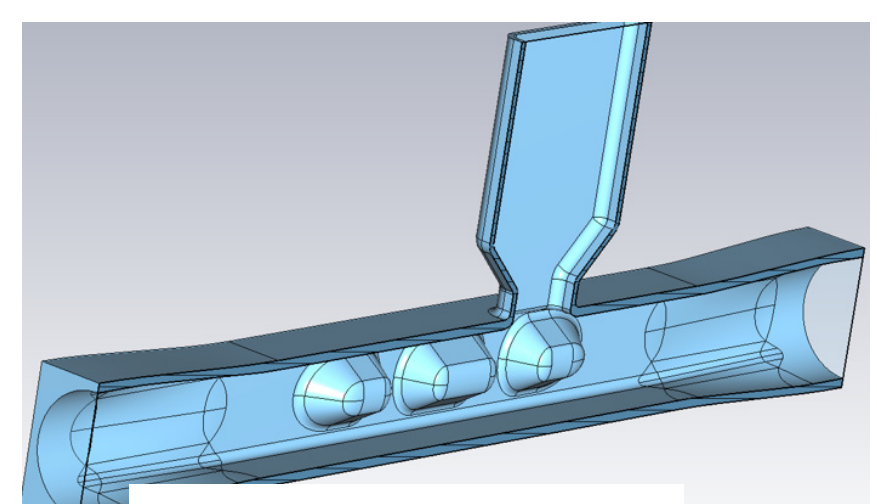

LFD at Kick $=0.9 \mathrm{MeV}$

Wall thickness $4 \mathrm{~mm}$.

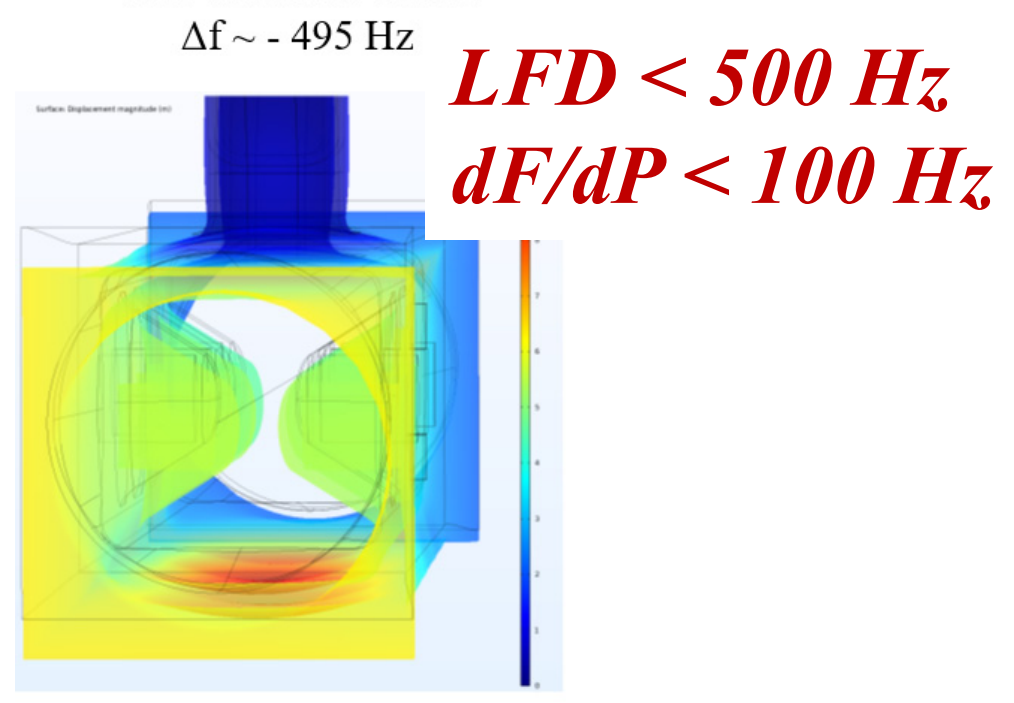

Deformation due to LFD

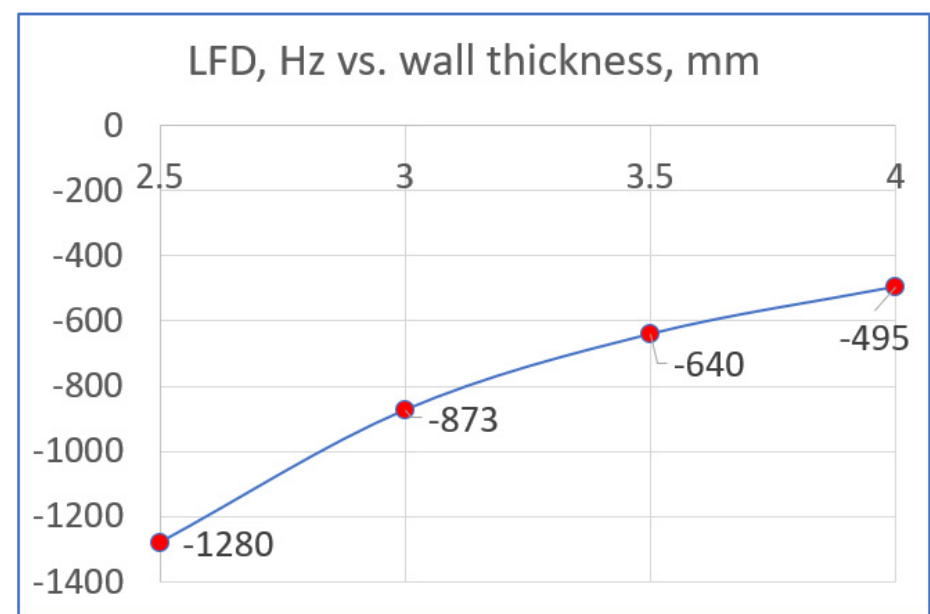

LFD in $\mathrm{Hz}$ at $\mathrm{Kick}=0.9 \mathrm{MeV}$ vs. cavity wall thickness

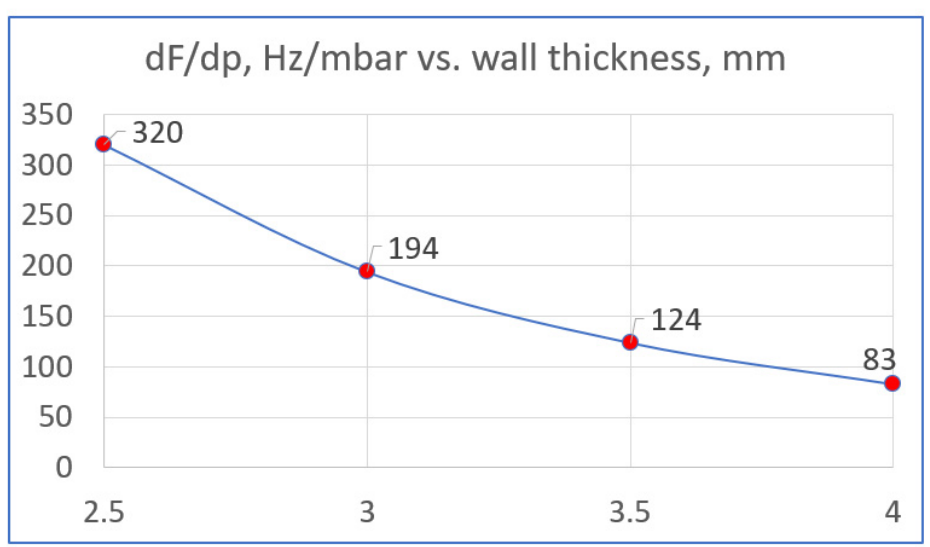

$\mathrm{df} / \mathrm{dP}$ in $\mathrm{Hz} / \mathrm{mbar}$ vs. cavity wall thickness

- QMiR LFD and dF/dP are less than the cavity bandwidth (few $\mathrm{kHz}$ ) 


\section{Mechanical Analysis of Frequency Tuning (by I. Gonin)}

Cavity shell deformations under external force. Wall thickness $4 \mathrm{~mm}$

$\Delta \mathrm{f} / \Delta \mathrm{L} \sim-45 \mathrm{kHz} / \mu \mathrm{m}$

$\Delta \sigma / \Delta$ Force $\sim 17.3 \mathrm{MPa} / \mathrm{kN}$
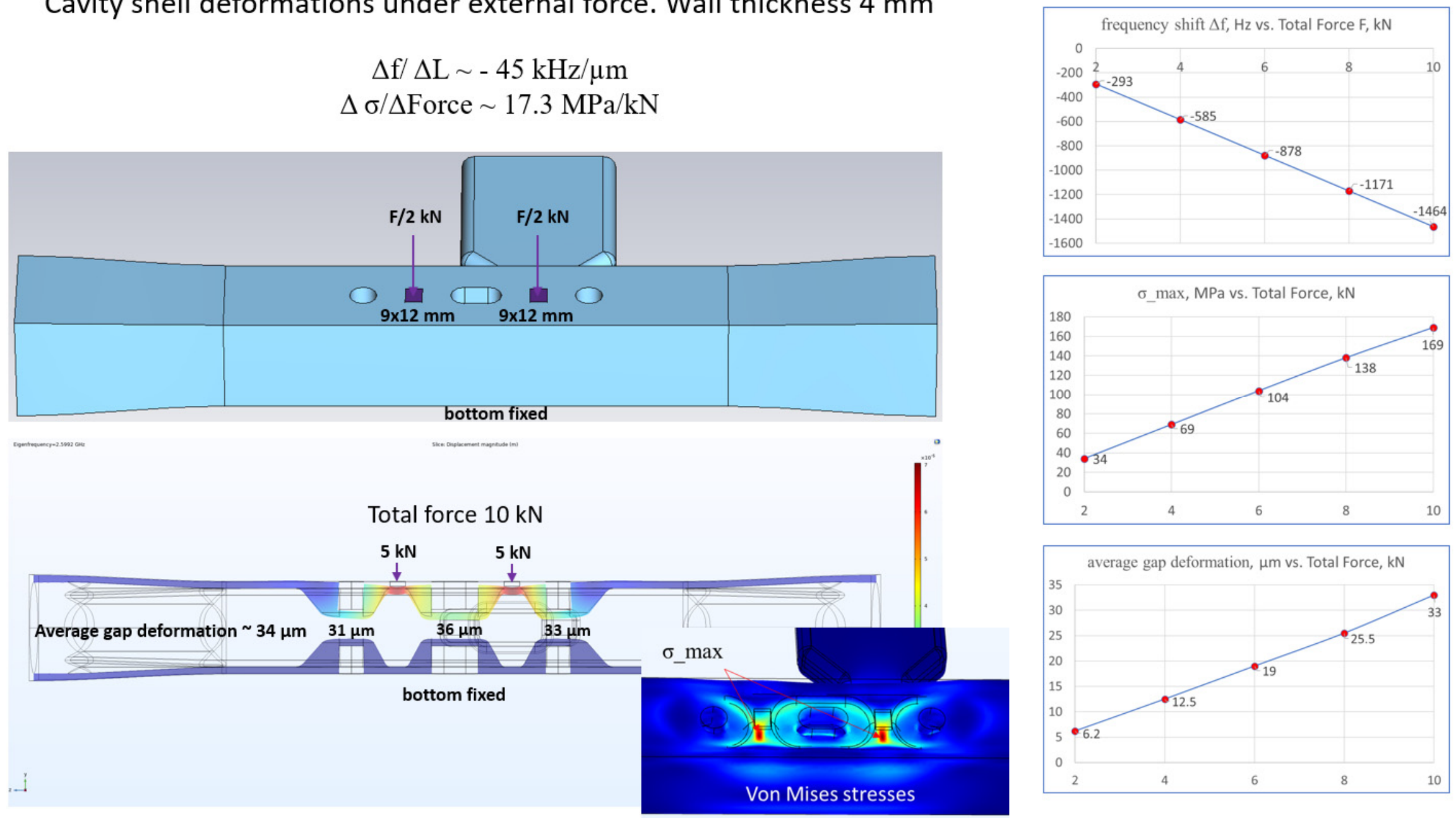

\section{Maximum frequency tuning range: $\sim 1 . .2 \mathrm{MHz}$}




\section{QMiR Cavity Slow Tuner Design (by V. Polubotko)}

Compact lever-type frequency tuner

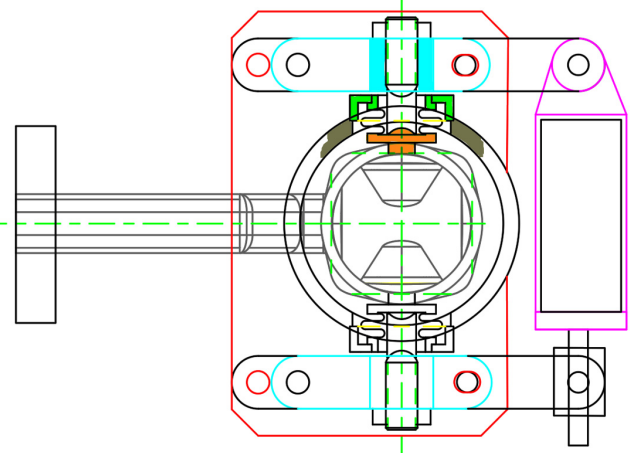

LHe Vessel

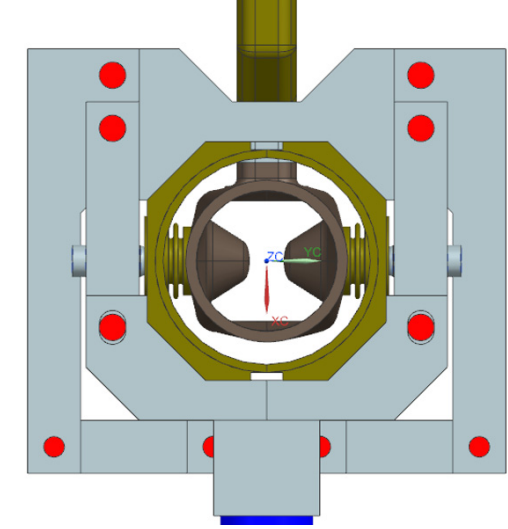

Dressed QMiR Cavity

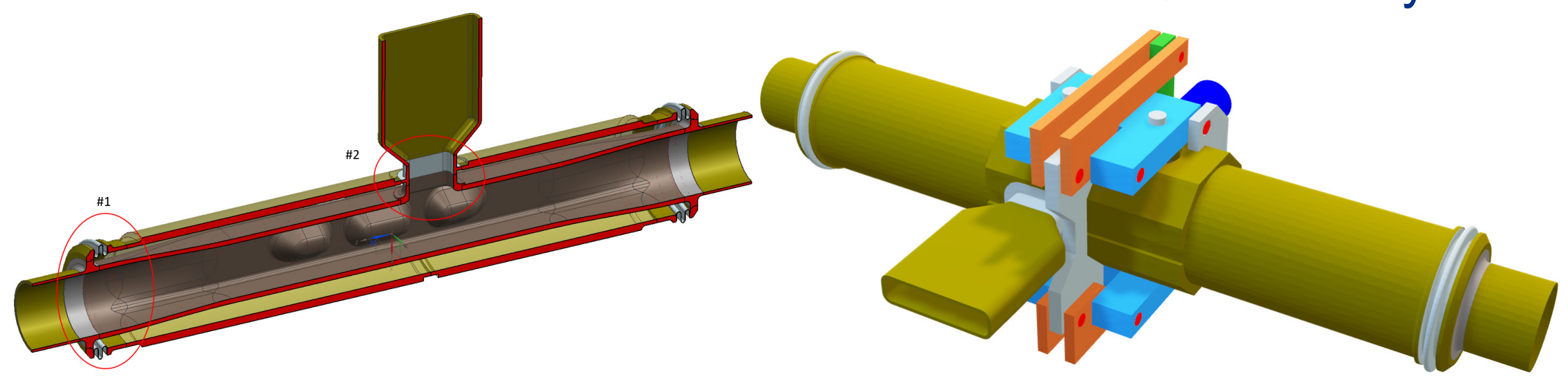

Design of frequency tuner integrated with dressed cavity is ongoing 華 Fermilab 


\section{Conclusions}

- Preliminary requirements for the ILC Crab Cavity developed

A A Quasi-Waveguide Multicell Deflecting Resonator (QMIR) is a good option for the ILC Crab Cavity

- QMIR is very compact and simple;

- It has sparse HOM spectrum;

- It has acceptable loss/kick factors;

- For the deflecting voltage of about $0.9 \mathrm{MV}$ the cavity has considerably small surface fields, $E_{p} \approx 25 \mathrm{MV} / \mathrm{m}, B_{\mathrm{p}} \approx 35 \mathrm{mT}$.

- No MP in operation voltage domain.

$\square$ QMIR cavity is considered now for Elletra-2, Trieste.

$\square$ Fermilab can design, build and test QMIR cavity for ILC application. 


\section{Backup Slides}

\section{EM design of the QMiR deflecting cavity}

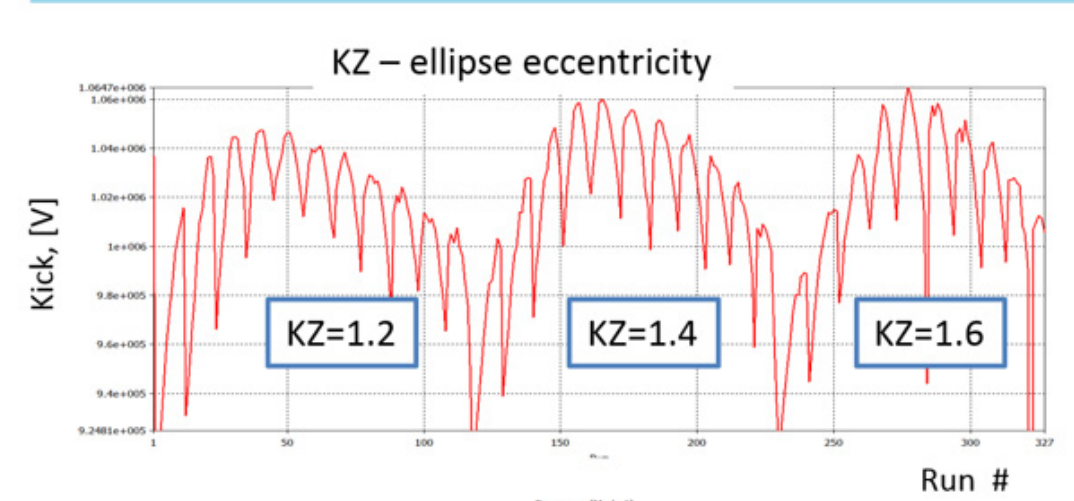

Operating trapped mode surface electric (up) and magnetic (down) fields

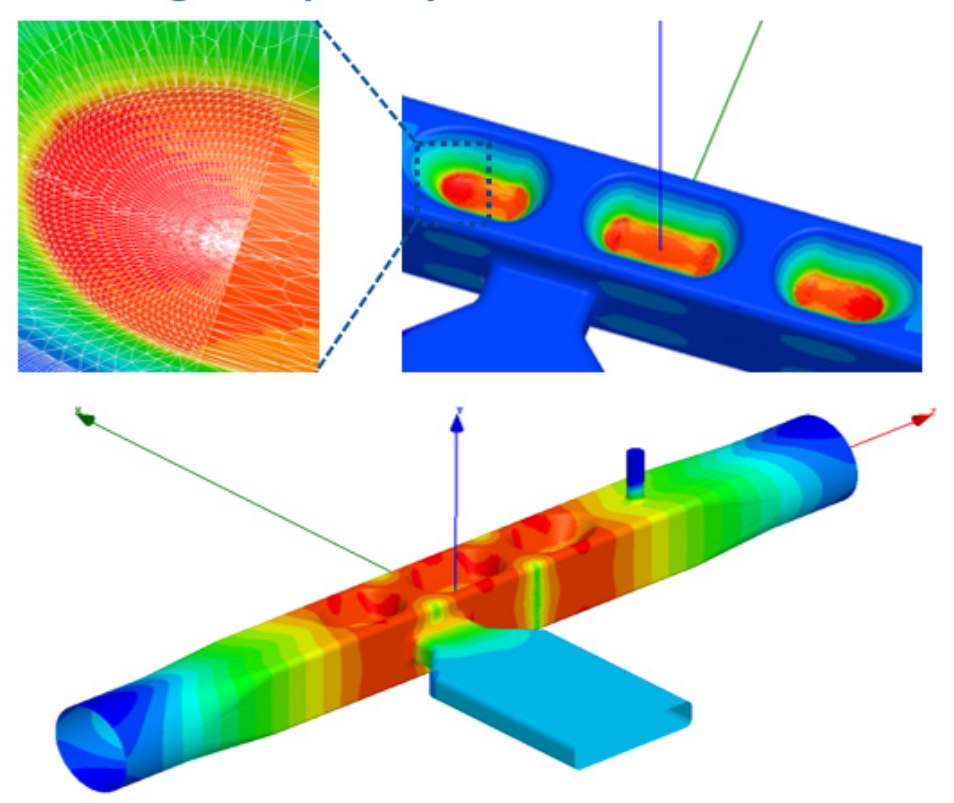

- Model is fully parameterized

- The frequency derivation was calculated for each parameter in order to preserve the operating mode frequency on the stage of geometry creation.

- General ellipsoid is used for hollow surface representation

- Global optimum search algorithm 


\section{Backup Slides}

\section{Same Order Mode (SOM) Damping}
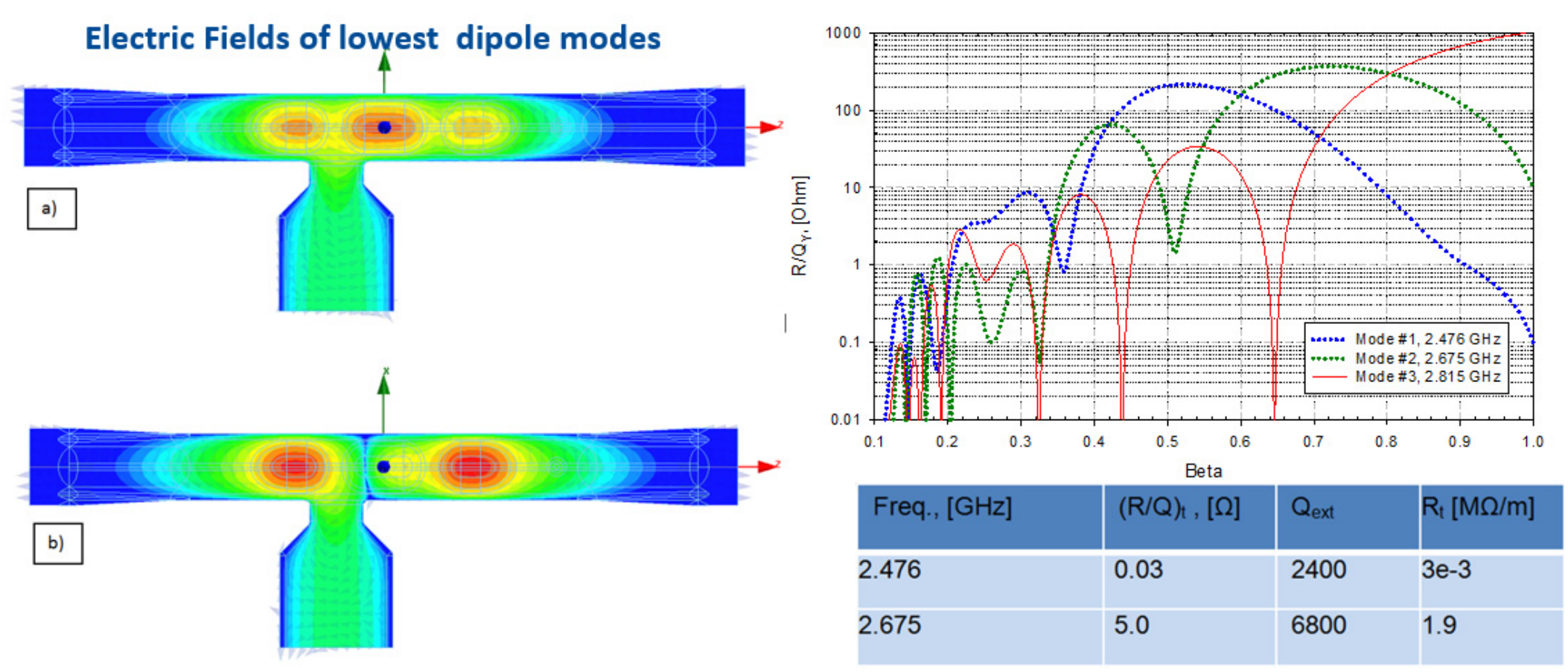

- The fundamental coupler waveguide is used to suppress SOM modes

- The FPC is purposely shifted from the cavity center in order to provide external coupling for the operating mode and damping lower frequency dipole modes simultaneously 


\section{Backup Slides}

\section{High Order Modes (HOM) Damping}

\section{Driven Modal Simulations}

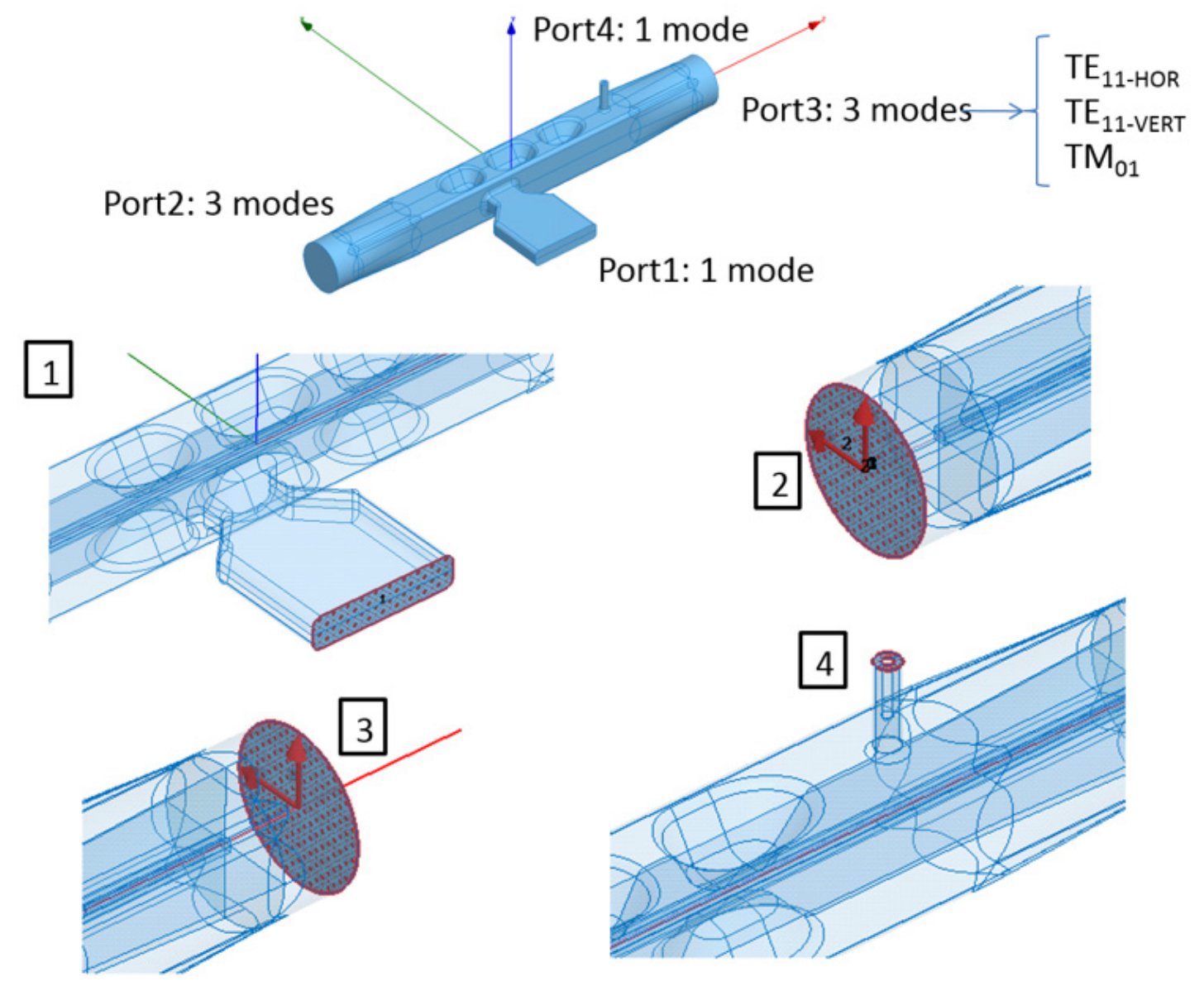




\subsection{GHz QMiR for ILC Crab Cavity}

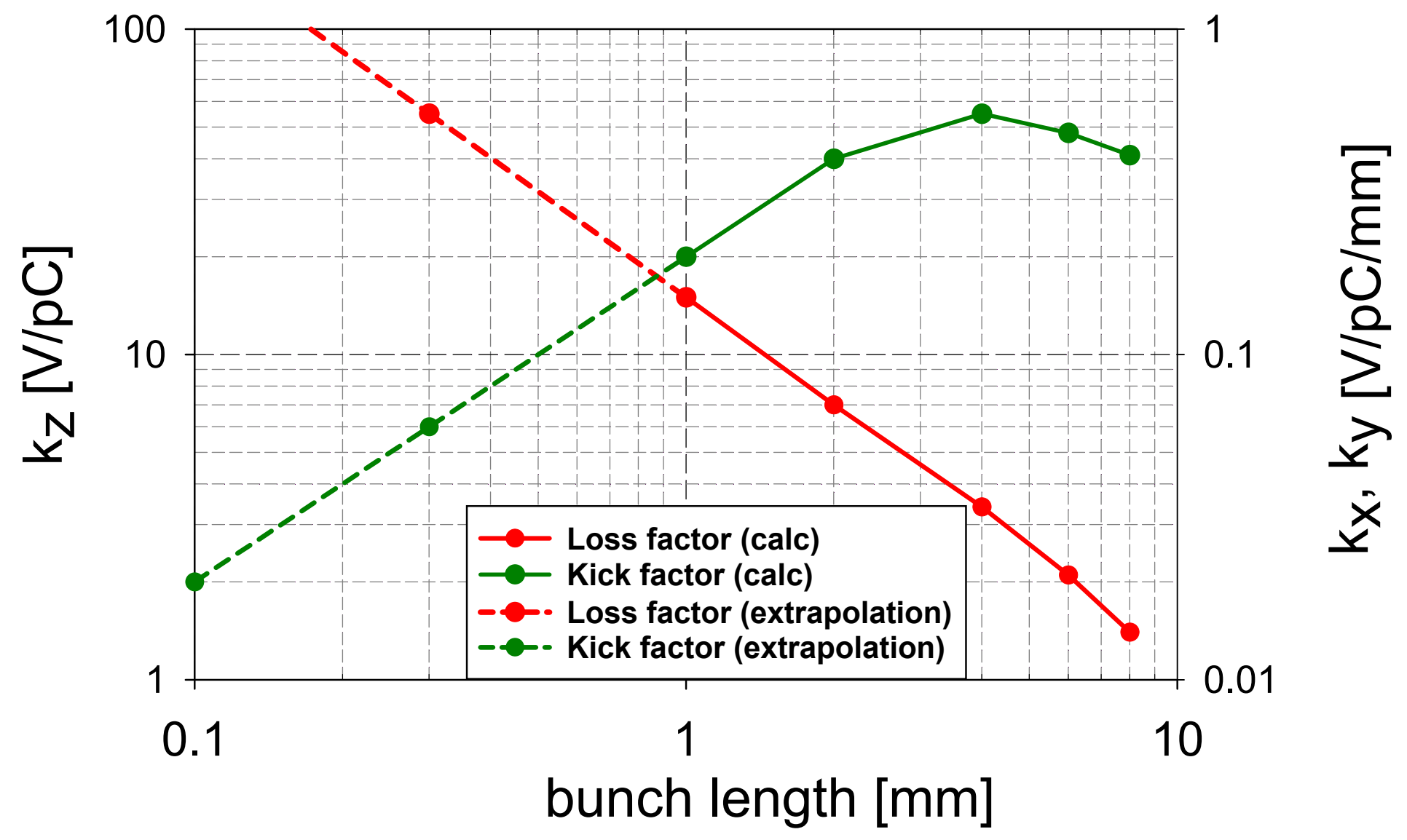

For the ILC bunch length $(0.3 \mathrm{~mm}$ rms), the loss and kick factors:

$\mathrm{k} \_$loss $<=50 \mathrm{~V} / \mathrm{pC}$ and $\mathrm{k}$ _kick $<=0.1 \mathrm{~V} / \mathrm{pC} / \mathrm{mm}$ 